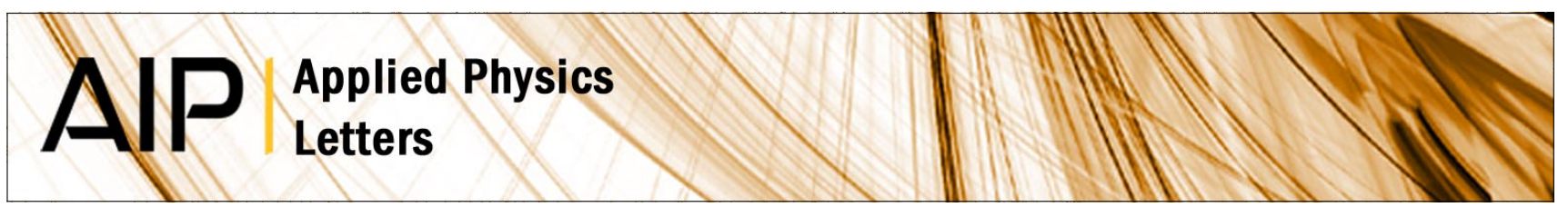

\title{
The SPARC linear accelerator based terahertz source
}

E. Chiadroni, A. Bacci, M. Bellaveglia, M. Boscolo, M. Castellano et al.

Citation: Appl. Phys. Lett. 102, 094101 (2013); doi: 10.1063/1.4794014

View online: http://dx.doi.org/10.1063/1.4794014

View Table of Contents: http://apl.aip.org/resource/1/APPLAB/v102/i9

Published by the American Institute of Physics.

\section{Related Articles}

A pepper-pot emittance meter for low-energy heavy-ion beams

Rev. Sci. Instrum. 84, 025117 (2013)

FLUTE: A versatile linac-based THz source

Rev. Sci. Instrum. 84, 022705 (2013)

Electron diffraction using ultrafast electron bunches from a laser-wakefield accelerator at $\mathrm{kHz}$ repetition rate Appl. Phys. Lett. 102, 064104 (2013)

Cooperative accumulation of coherent undulator radiation emitted from periodic electron bunches Phys. Plasmas 20, 014502 (2013)

New beam line for time-of-flight medium energy ion scattering with large area position sensitive detector Rev. Sci. Instrum. 83, 095107 (2012)

\section{Additional information on Appl. Phys. Lett.}

Journal Homepage: http://apl.aip.org/

Journal Information: http://apl.aip.org/about/about_the_journal

Top downloads: http://apl.aip.org/features/most_downloaded

Information for Authors: http://apl.aip.org/authors

\section{ADVERTISEMENT} \section{AIP $\begin{aligned} & \text { Applied Physics } \\ & \text { Letters }\end{aligned}$}

\section{EXPLORE WHAT'S NEW IN APL}

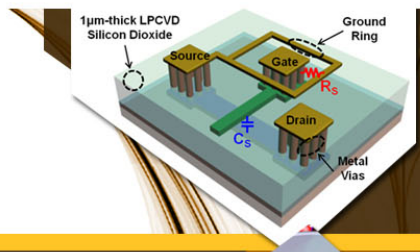

SURFACES AND INTERFACES

Focusing on physical, chemical, biological structural, optical, magnetic and electrical properties of surfaces and interfaces, and more.. 


\title{
The SPARC linear accelerator based terahertz source
}

\author{
E. Chiadroni, ${ }^{1}$ A. Bacci, ${ }^{1}$ M. Bellaveglia, ${ }^{1}$ M. Boscolo, ${ }^{1}$ M. Castellano, ${ }^{1}$ L. Cultrera, ${ }^{1}$ \\ G. Di Pirro, ${ }^{1}$ M. Ferrario, ${ }^{1}$ L. Ficcadenti, ${ }^{1}$ D. Filippetto, ${ }^{1}$ G. Gatti, ${ }^{1}$ E. Pace, ${ }^{1}$ A. R. Rossi, ${ }^{1}$ \\ C. Vaccarezza, ${ }^{1}$ L. Catani, ${ }^{2}$ A. Cianchi, ${ }^{2}$ B. Marchetti, ${ }^{2}$ A. Mostacci, ${ }^{3}$ L. Palumbo, ${ }^{3}$ \\ C. Ronsivalle, ${ }^{4}$ A. Di Gaspare, ${ }^{5}$ M. Ortolani, ${ }^{5}$ A. Perucchi, ${ }^{6}$ P. Calvani, ${ }^{7}$ O. Limaj, ${ }^{7}$ \\ D. Nicoletti, ${ }^{7}$ and S. Lupi ${ }^{7}$ \\ ${ }^{1}$ INFN-LNF, Frascati, Italy \\ ${ }^{2} I N F N$ and University of Rome "Tor Vergata," Rome, Italy \\ ${ }^{3}$ INFN and SBAI Department, Sapienza University of Rome, Rome, Italy \\ ${ }^{4}$ ENEA C.R. Frascati, Italy \\ ${ }^{5}$ Institute for Photonics and Nanotechnology, CNR-IFN Rome, Italy \\ ${ }^{6}$ Elettra Sincrotrone Trieste, Trieste, Italy \\ ${ }^{7}$ INFN and Department of Physics, Sapienza University of Rome, Rome, Italy
}

(Received 10 May 2012; accepted 15 February 2013; published online 4 March 2013)

\begin{abstract}
Ultra-short electron beams, produced through the velocity bunching compression technique, are used to drive the SPARC linear accelerator based source, which relies on the emission of coherent transition radiation in the terahertz range. This paper reports on the main features of this radiation, as terahertz source, with spectral coverage up to $5 \mathrm{THz}$ and pulse duration down to $200 \mathrm{fs}$, with an energy per pulse of the order of several micro-joule, and as electron beam longitudinal diagnostics.
\end{abstract} (C) 2013 American Institute of Physics. [http://dx.doi.org/10.1063/1.4794014]

In recent years, a huge experimental effort has been produced to fill the so-called THz gap. Indeed this spectral range, roughly located between the infrared and the microwave region $(0.1-10 \mathrm{THz})$, has been poorly investigated so far mainly because of the lack of intense and stable $\mathrm{THz}$ sources. The possibility of performing $\mathrm{THz}$ pump-probe spectroscopy is still practically unexplored. The main difficulty lies in obtaining electric fields from $100 \mathrm{kV} / \mathrm{cm}$ to $1 \mathrm{MV} / \mathrm{cm}$, corresponding approximately to the values at which the THz field becomes useful as a pump-beam. An effective $\mathrm{THz}$ source, hence, should have a high peak field, a spectral coverage up to $10 \mathrm{THz}$, and a full pulse shaping. ${ }^{1-3}$ Several technologies have been implemented worldwide to produce $\mathrm{THz}$ radiation. These technologies span from semiconductor-based photoconductive antennas to optical rectification in non-linear crystals. Resonant structures based on photonic crystals have been proposed as well in order to improve $\mathrm{THz}$ generation efficiency. ${ }^{4}$

In this paper, we report on the broadband $\mathrm{THz}$ radiation produced at the SPARC test facility ${ }^{5}$ by relativistic, short (sub-ps) electron bunches passing through the interface between different refractive index media, ${ }^{6,7}$ i.e., the socalled coherent transition radiation (CTR). The sub-ps regime is obtained at SPARC through the innovative velocity bunching (VB) technique. ${ }^{8,9}$ The spectral emission has been measured as a function of different machine operating conditions and compared to theoretical calculations. Experimental results presented here set the possibility to use the SPARC $\mathrm{THz}$ source for performing experiments in different fields. In particular, the high peak power (several MW) and the short pulse duration (few hundreds of $\mathrm{fs}$ ), measured under conservative electron beam parameters, i.e., low charge and moderate compression factors, make the SPARC THz source suitable to perform $\mathrm{THz}$ pump-probe experiments. In addition, coherent $\mathrm{THz}$ radiation can be a valid tool for electron beam longitudinal diagnostics. ${ }^{10}$
The spectral angular distribution produced by a relativistic electron beam can be written as ${ }^{11}$

$$
\frac{d^{2} I}{d \omega d \Omega}=\frac{d^{2} I_{s p}}{d \omega d \Omega}[N+N(N-1) F(\omega)],
$$

where $\omega=2 \pi c / \lambda$ is the radiation angular velocity, $N$ is the number of particles in the bunch, $\frac{d^{2} I_{s p}}{d \omega d \Omega}$ is the spectral angular distribution for a single particle, which depends on the specific emission mechanism. Under general conditions, $F(\omega)$ is the so-called three-dimensional bunch form factor, ${ }^{12}$ defined as the square of the Fourier transform of the normalized particle distribution within the bunch, $S(\vec{r})$,

$$
F(\omega)=\left|\int_{V} S(\vec{r}) e^{-i \frac{i}{c} \hat{n} \cdot \vec{r}} d \vec{r}\right|^{2} .
$$

Here, $\hat{n}$ is the unit vector towards the observation point, and the integral is performed over the bunch volume $V .^{13-15}$ The phase $\phi=\frac{\omega}{c} \hat{n} \cdot \vec{r}$ in Eq. (2) can be written, in cylindrical coordinates, as $\phi=\frac{\omega}{c}(\rho \sin \vartheta+z), \rho$ being the beam radius and $z$ the longitudinal coordinate with respect to the bunch center of mass; $\vartheta$ is the observation angle. As a consequence, at small observation angles, typical for radiation from highly relativistic electrons, and/or for finely collimated beams, the bunch transverse form factor has a rather weak influence on the CTR frequency spectrum. ${ }^{16}$ In the following, we refer to $F(\omega)=$ $\left|\int S(z) e^{-i \frac{i \omega}{c} z} d z\right|^{2}$ as the bunch longitudinal form factor, the contribution of the transverse one being negligible in the cases reported in the paper. The bunch longitudinal form factor is typically different from zero for wavelengths of the order, or longer than the bunch length, $\sigma_{z}=c \sigma_{t}$; therefore, the term in Eq. (1), proportional to $N^{2}$, dominates the emission, resulting in a coherent radiation, generally in the microwaves. The spectral range in which coherent emission takes place can be extended towards high frequency by compressing the bunch 
longitudinally. Since the coherent part of the spectrum is strongly enhanced by its $N^{2}$ dependence, the intensity gain with respect to most existing $\mathrm{THz}$ sources can be huge. As short electron bunches are mandatory for the generation of high peak $\mathrm{THz}$ power, different bunch compression techniques have been proposed. In circular machines ${ }^{17-19}$ however, due to their intrinsic magnetic optics limitation, only the ps-regime can be achieved, providing a spectral coverage up to nearly $1 \mathrm{THz}$. The sub-ps regime, corresponding to a spectral coverage up to several THz, can be reached, instead, in linear accelerator (linac) based machines, where either radio frequency $(\mathrm{RF})$ or magnetic compression can be performed.

At SPARC, the longitudinal compression is performed by using RF compression, the so-called VB technique. ${ }^{20}$ The VB method is based on the time-velocity correlation in an electron bunch, causing electrons in the bunch tail to be faster than electrons in the bunch head. The correlated chirp induces a longitudinal phase space rotation in the traveling RF wave potential (longitudinal focusing) accelerating the beam inside a long multi-cell RF structure. Thus simultaneously, an off crest energy chirp is applied to the injected beam. If the beam is slightly slower than the phase velocity of the RF wave, when injected at the zero crossing field phase, it slips back to phases where the field is accelerating and, simultaneously, it is chirped and compressed. Compression and acceleration take place at the same time within the same accelerating section, i.e., the first one following the SPARC gun. Longitudinal and transverse phase space distortions, characteristic of the VB technique, lead to asymmetric current profiles and higher final projected emittances, which can be optimized by applying proper emittance compensation schemes. ${ }^{21}$ The asymmetric longitudinal profile is instead responsible of the emission at higher frequencies, thanks to the sharp rising charge distribution at the bunch head.

SPARC is based on a 1.6 cell RF gun operating at S-band $(2.856 \mathrm{GHz}$, of the BNL/UCLA/SLAC type) and with high-peak field $(120 \mathrm{MV} / \mathrm{m})$ on the embedded copper photocathode, generating a $5.6 \mathrm{MeV}$ electron beam ${ }^{22}$ at $10 \mathrm{~Hz}$ repetition rate. The beam is then focused and matched into three traveling wave accelerating sections, which boost its energy up to $170 \mathrm{MeV}$. The first two sections are embedded in solenoids in order to provide additional magnetic focusing to better control the beam envelope and the emittance oscillations under VB schemes. ${ }^{21}$ At the end of the linac, a diagnostics section allows to characterize the accelerated beam through both transverse ${ }^{23}$ and longitudinal measurements. ${ }^{24}$ The diagnostic transfer line, equipped with quadrupoles and an RF deflecting cavity (RFD), ${ }^{25}$ is also used to match the beam to the SPARC undulator (in the main line) for free electron laser (FEL) experiments ${ }^{26,27}$ or, through bending magnets, to the by-pass line for THz radiation experiments. ${ }^{28}$ Figure 1 shows a sketch of the SPARC layout.

The THz station is placed at the end of the by-pass line. CTR is generated by the electron beam traversing the interface between the vacuum and an aluminum coated silicon screen placed in the vacuum pipe at $45^{\circ}$ with respect to the electron beam direction. Backward CTR is extracted through a z-cut quartz window, transported in air, and focused by means of a couple of twin $90^{\circ}$ off-axis parabolic mirrors onto a $\mathrm{THz}$ detector. Customized band-pass $\mathrm{THz}$ filters with a

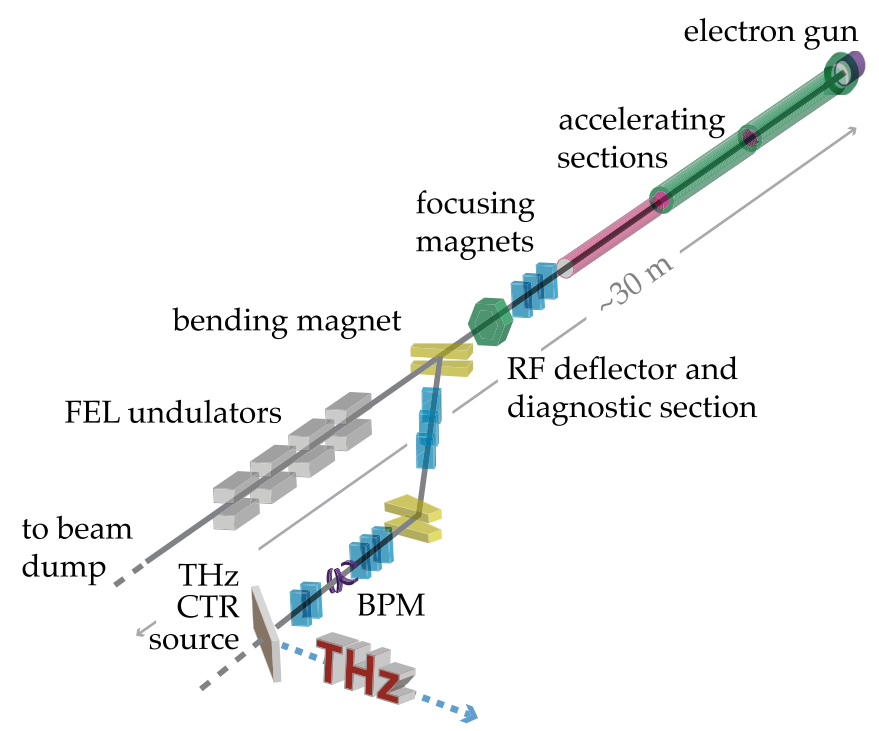

FIG. 1. Schematic layout of the SPARC test facility.

factor of merit $Q=10$, mounted on a remotely controlled filter wheel, can be inserted in front of the detector and used to select specific spectral regions. These are centered around the atmosphere-THz transparency-windows, i.e., $0.38-1.5-2.5$ $-3.4-4.3-4.8 \mathrm{THz}$. They are built up on high-resistivity silicon wafers through the electron beam lithographic technique. ${ }^{29}$ The detector can be remotely adjusted both along the optical axis (z) and in the transversal plane (x,y), allowing the fine adjustment of the focal plane and a transversal mapping of the source. Either pyroelectric and Golay cell detectors, both operating in a spectral range of $0.1-10 \mathrm{THz}$, have been used. The responsivity of both detectors, as provided by manufacturers, has been further checked at $270 \mathrm{GHz}$ by measuring, with the same THz optical set-up, the $1 \mathrm{~mW} \mathrm{THz} \mathrm{signal}$ from a Gunn oscillator by Virginia-Diode, Inc. The finite size of the screen (i.e., $30 \mathrm{~mm} \times 30 \mathrm{~mm}$ ), ${ }^{32}$ the vacuum chamber geometry (i.e., $63 \mathrm{~mm}$ vacuum window clear aperture, at $70 \mathrm{~mm}$ distance from the target, and $60 \mathrm{~mm}$ beam pipe radius) and the optics acceptance introduce a low frequency cut-off estimated to be $100 \mathrm{GHz}$. At high frequencies, the z-cut crystalline quartz window allows transmission up to $5 \mathrm{THz}$.

The SPARC THz source has been characterized under different beam charge values and bunch compression factors, in order to investigate different emission regimes. The beam energy varies from $167 \mathrm{MeV}$ to $102 \mathrm{MeV}$, depending on the injection phase in the linac first section, the one used as RF bunch compressor in the VB regime. When the beam is injected into the linac first section at the phase of the maximum RF field, it is accelerated on the crest of the RF field; therefore the beam gains the maximum energy and it is not longitudinally compressed.

The coherent emission has been studied and characterized by measuring the CTR signal as a function of the bunch charge $Q$.

A first set of measurements has been done in conditions of no RF longitudinal compression for different beam charge values in the range $130-470 \mathrm{pC}$. Due to the effect of longitudinal space charge forces, a bunch lengthening occurs with the increase of charge as measured with the RFD and shown in Fig. 2(a). Experimental data are fitted by the function 

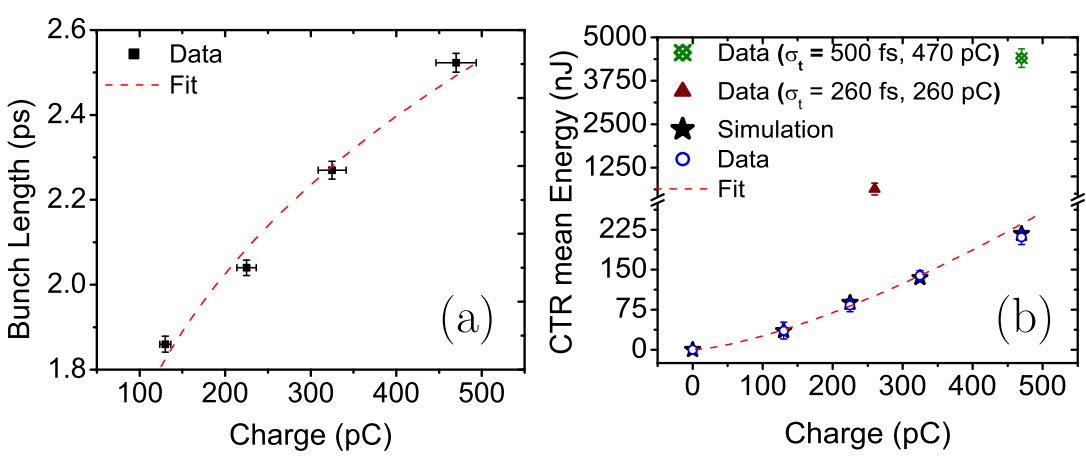

FIG. 2. (a) RMS bunch length (black squares) measured as function of the bunch charge. The red dashed line is the fit function $\sigma_{t}(Q) \propto Q^{B}$, with $B=0.243$ (0.010). (b) CTR energy as function of the charge (data, blue circles and calculation, black stars). The fit (red dashed curve) of experimental data provides a power law of $Q^{b}$, with $b=1.44(0.33)$. Two more data points, olive crossed diamond ( $500 \mathrm{fs}$ and $470 \mathrm{pC}$ ) and purple triangle (260 fs and $260 \mathrm{pC}$ ), highlight the effect of sub-ps bunches on CTR energy.

$$
\sigma_{t}(Q)=A Q^{B}
$$

with $A=0.560$ (0.032) $s / C^{B}$ and $B=0.243$ (0.010).

The $\mathrm{THz}$ emission was affected by the bunch charge even through the form factor because of the slightly different bunch length. Indeed, assuming a Gaussian longitudinal beam profile (as experimentally observed in this set of measurements),

$$
S(t)=\frac{e^{-t^{2} / 2 \sigma_{t}^{2}}}{\sqrt{2 \pi} \sigma_{t}} \leftrightarrow F(\omega)=e^{-\omega^{2} A^{2} Q^{2 B}},
$$

according to Eq. (3). The integrated CTR spectral angular distribution can be analytically computed on the $\omega$ axis as

$$
I_{C T R}=\int_{\Delta \Omega} \int_{0}^{\infty} \frac{d^{2} I}{d \omega d \Omega} d \omega d \Omega \propto Q^{2-B},
$$

because of the Gaussian shape of $F(\omega)$. B is the power estimated from the law describing the bunch length variation as function of the bunch charge (Eq. (3)). The assumption of infinite screen size allows to consider $\frac{\dot{d}^{2} I_{s p}}{d \omega d \Omega}$ independent of the frequency and does not affect the dependence on the charge.

The measured CTR energy (blue circles) is shown in Fig. 2(b) as a function of the charge. Experimental data are compared with a calculation (black stars) performed with the THz Transport code, ${ }^{33}$ where the beamline (i.e., mirrors, detector, and quartz window) has been taken into account. The red dashed curve is a $a Q^{b}$ fit with $b=1.44$ (0.33): this behavior is in good agreement, within the error bar, with the numerical power law $(2-B)=1.757(0.010)$, with $B=0.243$ (0.010), taking into account the effect of bunch lengthening for an increasing charge. This effect explains the deviation from the quadratic law expected from the theory strictly valid for constant bunch length.

A second set of measurements has been done by operating the SPARC linac in RF compression mode: the two points represented by an olive crossed diamond and a purple triangle in Fig. 2(b) have been measured in this regime and correspond to a charge of $470 \mathrm{pC}$ and $260 \mathrm{pC}$, respectively. Comparing the $\mathrm{THz}$ emission at high charge $(470 \mathrm{pC})$, for the uncompressed beam (2.523(0.022) ps pulse duration) with the case of a 500 fs compressed beam, it is evident that a compression factor of 5 allowed to gain a factor 20 in CTR energy, passing from 211(13) nJ measured with the long bunch to 4402(264) nJ achieved with compression. With the second working point (at $260 \mathrm{pC}$ ), the lower charge value allowed us to rise the compression factor to 8 passing from a beam length of $2.044(0.020)$ ps (on crest operation) to 260(5) fs rms, whose temporal profile, as measured with the RFD, is shown in Fig. 3(a).

The sharper current profile results in a wider $\mathrm{THz}$ spectral coverage, extending up to the limit of our system, i.e., $5 \mathrm{THz}$. The CTR energy per frequency unit has been measured at different frequencies by customized band-pass filters. ${ }^{29}$ The Golay cell frequency response and the filters transmission have been taken into account to retrieve the measured CTR energy spectrum shown in Fig. 3(b), where data (red squares) are also compared to calculations (solid olive and dashed blue lines). The dashed blue curve corresponds to the CTR energy density as obtained through the ideal Ginzburg-Frank (GF) formula, while the olive solid one has been calculated through the generalized GF equation. ${ }^{30}$ In both cases, the drop above $5 \mathrm{THz}$ is mainly caused by the longitudinal form factor of the bunch. The drop of the solid olive curve below $300 \mathrm{GHz}$ depends on the finite dimension of the screen with respect to the source size. This effect is not taken into account in the ideal GF calculation which shows a nearly constant CTR energy at low frequency. A very good agreement is found at higher frequencies among data and calculations (Fig. 3(b)), while a discrepancy is observed in the lower part of the spectrum. This discrepancy is mainly due to diffraction effects in the collection optics and window clear aperture which are not considered in the generalized GF calculation. ${ }^{31}$ The corresponding CTR mean

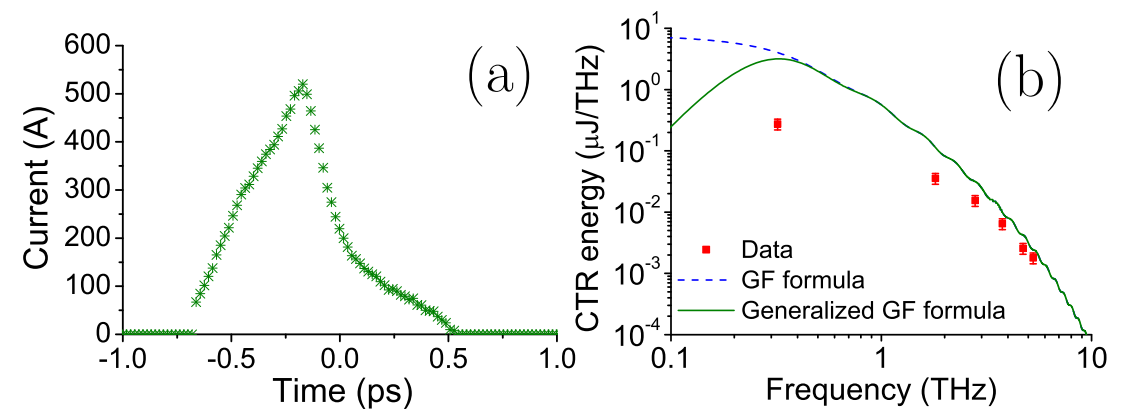

FIG. 3. (a) Longitudinal bunch profile measured with the RFD in case of a 260 fs RMS bunch duration with $260 \mathrm{pC}$ charge. (b) Corresponding measured CTR pulse energy density in $\mu \mathrm{J} / \mathrm{THz}$ as function of frequency (red squares). Dashed blue and solid olive curves correspond to the CTR pulse energy density calculated from the ideal GF formula and the generalized one, respectively, taking into account the measured form factor. 

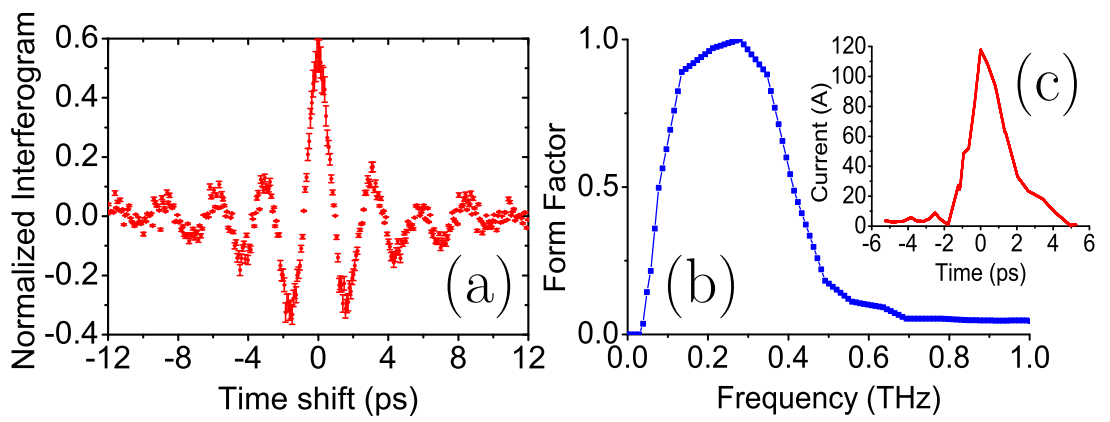

FIG. 4. (a) CTR autocorrelation function as measured through a Martin-Puplett interferometer. (b) Bunch form factor and, (c) the retrieved longitudinal bunch profile, providing a RMS bunch length of 1.4(0.10) ps.

energy measured in the whole experimental frequency range, i.e., $150 \mathrm{GHz}-5 \mathrm{THz}$, is reported in Fig. 2(b) (purple triangle), i.e., 635(70) nJ.

The characterization of the SPARC longitudinal density profile under moderate compression has been performed using a frequency-domain technique based on the autocorrelation of CTR. The autocorrelation function of the CTR, produced by a 300 pC, $120 \mathrm{MeV}$ beam, is shown in Fig. 4(a), as measured using a Martin-Puplett interferometer. ${ }^{34}$ A detailed description of the layout can be found in Ref. 35. Due to the low and high frequency suppression, introduced by the experimental apparatus, only a portion of the CTR spectrum contributes to the reconstruction of the longitudinal bunch profile. Furthermore, since only the square of the Fourier transform of the longitudinal beam profile is evaluated, no information on the phase can be retrieved. Therefore, the reconstruction procedure of the bunch profile, based on Kramers-Kronig dispersion relation ${ }^{36,37}$ needs to be applied, allowing to determine the phase value consistent with the measured modulus. ${ }^{38}$ The accuracy of the method increases extending the measured radiation spectrum. Therefore, it implies the knowledge of the transfer function of the experimental apparatus, i.e., screen size, transmission of vacuum window, interferometer transfer function, and the detectors frequency response. ${ }^{10}$ All these arguments are taken into account to reconstruct the bunch profile, shown in Fig. 4(c), which provides a RMS bunch length of 1.4(0.10) ps. The bunch form factor extends up to $0.5 \mathrm{THz}$ (Fig. 4(b)), in agreement with the (ps) long bunch.

In conclusion, ultra-short electron beams have been generated from the SPARC linac, taking advantages of the VB technique, and characterized both to provide a precise longitudinal diagnostics and to produce high peak power broadband $\mathrm{THz}$ radiation. The source spectral coverage extends up to several $\mathrm{THz}$ with energy per pulse larger than $4 \mu \mathrm{J}$ and an integrated peak power of the order of a few MW. An estimate of the electric field associated to the so generated $\mathrm{THz}$ pulses provides a value on the order of $700 \mathrm{kV} / \mathrm{cm} .{ }^{39}$ The planned increase in the machine energy and the possibility, given by the versatility of the VB technique, to explore innovative beam manipulation schemes ${ }^{40}$ may further increase the performances of the SPARC THz radiation opening the possibility to perform THz-pump THz-probe experiments.

${ }^{1}$ M. S. Sherwin, C. A. Schmuttenmaer, and P. H. Bucksbaum, "Opportunities in THz science," Report of a DOE-NSF-NIH Workshop (2004). Available at: http://science.energy.gov/ /media/bes/pdf/reports/files/thz_rpt.pdf.

${ }^{2}$ D. Daranciang, J. Goodfellow, M. Fuchs, H. Wen, S. Ghimire, D. A. Reis, H. Loos, A. S. Fisher, and A. M. Lindenberg, Appl. Phys. Lett. 99, 141117 (2011).
${ }^{3}$ M. C. Hoffmann, S. Schulz, S. Wesch, S. Wunderlich, A. Cavalleri, and B. Schimdt, Opt. Lett. 36(23), 4473-4475 (2011).

${ }^{4}$ Y.-S. Lee, Principles of Terahertz Science and Technology, (Springer, 2008).

${ }^{5}$ D. Alesini, S. Bertolucci, M. E. Biagini, C. Biscari, R. Boni, M. Boscolo, M. Castellano, A. Clozza, G. Di Pirro, A. Drago et al., Nucl. Instrum. Methods Phys. Res. A 507, 345-349 (2003).

${ }^{6}$ J. D. Jackson, Classical Electrodynamics, 3rd ed. (Wiley, 1995).

${ }^{7}$ Y. Shibata, K. Ishi, T. Takahashi, T. Kanai, M. Ikezawaet, K. Takami, T. Matsuyama, K. Kobayashi, and Y. Fujita, Phys. Rev. A 45, R8340 (1992).

${ }^{8}$ B. Aune and R. H. Miller, SLAC-PUB Report-2393, 1979.

${ }^{9}$ B. E. Carlsten, Nucl. Instrum. Methods Phys. Res. A 285, 313-319 (1989).

${ }^{10}$ E. Chiadroni, TESLA-FEL Report 2006-09 (2006).

${ }^{11}$ J. S. Nodvick and D. S. Saxon, Phys. Rev. 96, 180 (1954).

${ }^{12}$ R. Lai and A. J. Sievers, Nucl. Instrum. Methods Phys. Res. A 397, 221231 (1997).

${ }^{13}$ A. P. Potylitsyn, M. I. Ryazanov, M. N. Strikhanov, and A. A. Tischchenko, Diffraction Radiation from Relativistic Particles (SpringerVerlag, Berlin, 2010).

${ }^{14}$ S. Casalbuoni, B. Schmidt, and P. Schmueser, TESLA Report 2005-15 (2005).

${ }^{15}$ Y. Shibata, T. Takahashi, T. Kanai, K. Ishi, M. Ikezawa, J. Ohkuma, S. Okuda, and T. Okada, Phys. Rev. E 50, 1479 (1994).

${ }^{16}$ O. Grimm, H. D. Hashemi, J. Rossbach, V. Balandin, and N. Golubeva, TUPC030, Proc. of EPAC08, Genoa, Italy, 2008.

${ }^{17}$ M. Abo-Bakr, J. Feikes, K. Holldack, G. Wuestefeld, and H.-W. Huebers, Phys. Rev. Lett. 88, 254801 (2002).

${ }^{18}$ A.-S. Muller, Rev. Accel. Sci. Technol. 03(01), 165-183 (2010).

${ }^{19}$ S. Lupi, A. Nucara, A. Perucchi, P. Calvani, M. Ortolani, L. Quaroni, and M. Kiskinova, J. Opt. Soc. Am. B 24-4, 959-964 (2007).

${ }^{20}$ L. Serafini and M. Ferrario, AIP Conf. Proc. 581, 87-106 (2000).

${ }^{21}$ M. Ferrario, D. Alesini, A. Bacci, M. Bellaveglia, R. Boni, M. Boscolo, M. Castellano, E. Chiadroni, A. Cianchi, L. Cultrera et al., Phys. Rev. Lett. 104, 054801 (2010).

${ }^{22}$ M. Ferrario, D. Alesini, A. Bacci, M. Bellaveglia, R. Boni, M. Boscolo, M. Castellano, L. Catani, E. Chiadroni, S. Cialdi et al., Phys. Rev. Lett. 99, 234801 (2007).

${ }^{23}$ A. Mostacci, M. Bellaveglia, E. Chiadroni, A. Cianchi, M. Ferrario, D. Filippetto, G. Gatti, and C. Ronsivalle, Phys. Rev. ST Accel Beams 15, 082802 (2012).

${ }^{24}$ D. Filippetto, M. Bellaveglia, M. Castellano, E. Chiadroni, L. Cultrera, G. Di Pirro, M. Ferrario, L. Ficcadenti, A. Gallo, G. Gatti et al., Phys. Rev. ST Accel Beams 14(9), 092804 (2011).

${ }^{25}$ D. Alesini, G. Di Pirro, L. Ficcadenti, A. Mostacci, L. Palumbo, J. Rosenzweig, and C. Vaccarezza, Nucl. Instrum. Methods Phys. Res. A 568, 488 (2006)

${ }^{26}$ L. Giannessi, A. Bacci, M. Bellaveglia, F. Briquez, M. Castellano, E. Chiadroni, A. Cianchi, F. Ciocci, M. E. Couprie, L. Cultrera et al., Phys. Rev. Lett. 106, 144801 (2011).

${ }^{27}$ M. Labat, M. Bellaveglia, M. Bougeard, B. Carre', F. Ciocci, E. Chiadroni, A. Cianchi, M. E. Couprie, L. Cultrera, M. Del Franco et al., Phys. Rev. Lett. 107, 224801 (2011).

${ }^{28}$ E. Chiadroni, A. Bacci, M. Bellaveglia, P. Calvani, M. Castellano, A. Cianchi, G. Di Pirro, M. Ferrario, G. Gatti, O. Limaj et al., J. Phys.: Conf. Ser. 357, 012034 (2012).

${ }^{29}$ O. Limaj, A. Nucara, S. Lupi, M. Ortolani, A. Di Gaspare, E. Palange, and P. Carelli, Opt. Commun. 284, 1690-1693 (2011).

${ }^{30}$ S. Casalbuoni, B. Schmidt, P. Schmueser, V. Arsov, and S. Wesch, Phys. Rev. ST Accel Beams 12, 030705 (2009).

${ }^{31}$ G. R. Neil, G. L. Carr, J. F. Gubeli III, K. Jordan, M. C. Martin, W. R. McKinney, M. Shinn, M. Tani, G. P. Williams, and X.-C. Zhang, Nucl. 
Instrum. Methods Phys. Res. A 507(1-2), 537-540 (2003).

${ }^{32}$ M. Castellano, A. Cianchi, G. Orlandi, and V. A. Verzilov, Nucl. Instrum. Methods Phys. Res. A 435, 297-307 (1999).

${ }^{33}$ B. Schmidt, THzTransport code, private communication (2010).

${ }^{34}$ D. H. Martin and E. Puplett, Infrared Phys. 10, 105-109 (1970).

${ }^{35}$ E. Chiadroni, A. Bacci, M. Bellaveglia, M. Castellano, G. Di Pirro, M. Ferrario, G. Gatti, E. Pace, A. R. Rossi, C. Vaccarezza et al., J. Phys.: Conf. Ser. 359, 012018 (2012).
${ }^{36}$ R. Lai and A. J. Sievers, Phys. Rev. E 50, R3342 (1994).

${ }^{37}$ F. Wooten, Optical Properties of Solids (Academic, New York, 1972).

${ }^{38}$ R. Lai, U. Happek, and A. J. Sievers, Phys. Rev. E 50, R4294-R4297 (1994).

${ }^{39}$ S. L. Hulbert and G. P. Williams, Rev. Sci. Instrum. 80, 106103 (2009).

${ }^{40}$ M. Ferrario, D. Alesini, A. Bacci, M. Bellaveglia, R. Boni, M. Boscolo, P. Calvani, M. Castellano, E. Chiadroni, A. Cianchi et al., Nucl. Instrum. Methods Phys. Res. A 637, S43-S46 (2011). 